\title{
Where can Knowledge-Based Decision Support Systems Go in Contemporary Business Management - A New Architecture for the Future
}

\author{
Shaofeng Liu, Melanie Hudson Smith, Sarah Tuck, Jiang Pan, Ali Alkuraiji, and Uchitha Jayawickrama
}

\begin{abstract}
Decision support systems (DSS) are one of the most widely used management information systems in current business management. The focus of the paper is on the knowledge-based decision support systems, namely the KB-DSS in support of contemporary business management decision making. Business managers use KB-DSS can improve their decision making not only in terms of speed and accuracy but also consistency. Key perspectives of KB-DSS including technological, organizational, social and cultural perspectives are discussed in the context of contemporary management decision context. New contribution to the knowledge management function of KB-DSS through a number of recent projects is presented. The paper then highlights some implications for the development of the next generation of KB-DSS before a new architecture is proposed for future work.
\end{abstract}

Index Terms-Knowledge-based decision systems, knowledge levels, knowledge reuse, knowledge mobilization, critical knowledge, knowledge chain management, knowledge life cycle.

\section{INTRODUCTION}

Business management decisions have been known for a number of characteristics, for example, (1) their high significance such as a lot of money are involved and usually a lot of people's interests are at stake; (2) time limitation many business decisions have to be made within very tight time constraint in order for businesses to capture the market opportunity; (3) high degree of complexity - business decisions are often situated in rather complex environment which needs to consider simultaneously the product and service issues, supply chain issues, organizational issues, quality issues, cost-effectiveness etc. (4) high degree of uncertainty - business environment can change quickly, especially under the current unstable financial situation around the world, business decisions need to take account of this uncertainty in order to adapt to the evolving global market. Business managers have been facing great challenges in making the right decisions when dealing with decision situations that are extremely important, with high complexity and uncertainty, and under time pressure. As a result, business decision makers have been seeking for help from technologies such as IT over the past few decades in order to cope with the decision environment and make better decisions. Various types of decision support systems (DSS) have been developed and played an important role in

Manuscript received November 10, 2013; revised January 7, 2014.

The authors are with the Graduate School of Management, University of Plymouth, UK (e-mail: shaofeng.liu@plymouth.ac.uk). supporting business managers to improve business decisions [1].

The classic DSS architecture is typically comprised of three core components: a database management sub-system, a model base sub-system, and a user interaction management sub-system [2]. The DSS based on this classic architecture have solved the decision support issue for business managers to a certain extent: the DSS can provide the right information at the right time in the right format; the DSS can provide the right models with "what-if" analysis of decision alternatives; the DSS can provide effective interaction mechanisms such as decision dashboards so that information and analysis results can be presented to decision makers in an easy-to-understand manner. However, what these DSS lack is that the systems cannot provide domain knowledge and expertise to decision makers.

It has been acknowledged that the right decisions can only be reached based on the decision makers' good judgments, and good judgments are based on good knowledge [3]. As the scenarios a decision maker has to face are often complex, it is hardly possible for decision makers to have all the knowledge required to make those decisions without any help from outside sources such as agents (software systems) and shared work colleagues. Therefore, it is essential that decision makers seek-out knowledge through appropriate knowledge repository, reuse strategy and mechanisms. Furthermore, decision makers could benefit tremendously from easy to use knowledge systems that could equip them with extra expertise and knowledge about the decision problems and stimulate creative solutions to those problems [4]. To address the above issues, the concept of a new generation of DSS emerged in 1980s to include a knowledge management function and the systems were named as knowledge-based decision support systems (KB-DSS) [1], [5]-[7].

This paper will discuss some of the recent advancements in KB-DSS from different perspectives, especially in terms of the improvement of knowledge management function through multiple knowledge levels, knowledge reuse, knowledge mobilization, critical knowledge, knowledge chain management and knowledge integration. The next section will review related work in KB-DSS. Section III will present outputs from a number of research projects undertaken by the authors in terms of knowledge management to improve KB-DSS. Implications for the development of new KB-DSS for contemporary business decisions are put forward together with a new architecture for future work in Section IV. Finally, conclusions are drawn in Section V. 


\section{RELATED WORK}

The integration of the knowledge management function into classic DSS can improve decision making performance in two senses: (1) enhancing the quality of services by having an "expert" readily available to users when human experts are in short supply [4]; (2) assisting a human expert by making their decisions more consistently [8]. Since 1990s, knowledge management has been playing an important role in the new generation of DSS known as KB-DSS. In today's rapidly changing business world, agile and flexible organisations require their employees to frequently change their work focus. Therefore KB-DSS with domain knowledge can provide better support for decisions in general, and specifically through facilitating integration of decision models and decision processes (represented by expert advice, generating alternatives and choice of choices). Lying in the centre of knowledge management sub-system in KB-DSS are a knowledge base and an inference engine [1]. Fig. 1 shows the core components in a KB-DSS that have been widely accepted by scholars [5]-[7]. That is, a KB-DSS has an additional component including a knowledge base and an inference engine together with the three basic components from the classic DSS: a database management sub-system (DBMS), a model base management sub-system (MBMS) and a user interaction management sub-system which is often called a human-computer interface (HCI).

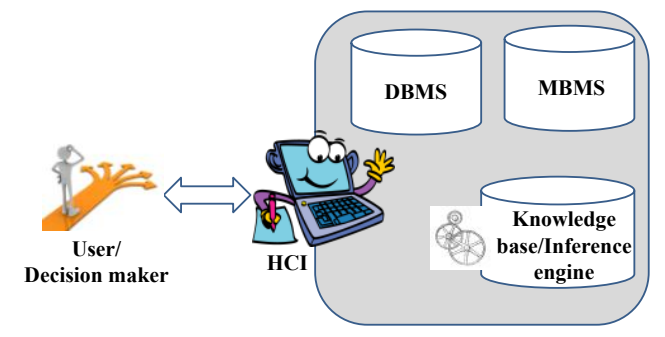

Fig. 1. Core components of KB-DSS.

KB-DSS has been mainly benefited from the advancement in Artificial Intelligence (AI) technology in the past few decades. AI technology has enabled the KB-DSS to have new functions such as better knowledge modeling and reasoning [9]. Many fully functioned knowledge-based systems (KBS) have been developed and used in business decision making as an independent system. However, some scholars have suggested that KB-DSS should have a KBS to be integrated into all three core components of a classic DSS, i.e. to include a knowledge management function in the traditional DBMS, MBMS and HCI of a DSS. For example, a knowledge management function can be integrated into the DBMS to help automatically update information and get rid of obsolete data. An MBMS with knowledge management has the ability to refine the decision models accordingly when the decision situation changes. Integration of a knowledge management function and an HCI will be able to have an intelligent decision interface.

Besides the knowledge base, the other important artifact in the knowledge management sub-system is the inference engine which has reasoning mechanisms. An inference engine is a piece of software programme that can refer to the knowledge in the knowledge base, manipulate the knowledge according to decision needs, and infer solutions and suggest actions to be taken [9]. Most inference engines can not only refer to knowledge in the knowledge base, but also can infer new knowledge when needed. They also decide which, when and the sequence existing knowledge can be activated. Recent work in KB-DSS in terms of its reasoning function can be classified around four important approaches: rule-based reasoning (RBR), case-based reasoning (CBR), network-based reasoning (NetBR) such as Bayesian networks and artificial neural networks, and narrative-based reasoning (NBR). RBR research has evolved significantly from the traditional "if-then" rule to the modern belief rule base which is capable of capturing vagueness, incompleteness and nonlinear causal relationships in many decision environments [10]. CBR systems do not need to construct decision rules, but are valuable examples of decision-support systems as they base their recommendations on the subset of the most similar or most reusable experiences previously encountered [11]. One of the main assets of CBR is its eagerness to learn. Learning in CBR can be as simple as memorizing a new case or can entail refining the memory organization or meta-learning schemes. There has been increasing use of NetBR in recent years. NetBR are probabilistic inference engines that can be used to reason under uncertainty. There is plenty of ongoing research on integrating NetBR into a wide range of decision making fields especially to solve complex semi-structured problems, for example [12]. While KB-DSS based on RBR, CBR and NetBR are mainly dealing with well-structured or semi-structured knowledge, in the knowledge-intensive industries such as social services, many business decisions require support from knowledge that are hidden in unstructured narratives such as clients' records and stories, from which NBR has emerged recently. Incorporating narratives can help people have a more comprehensible understanding of business decision challenges through listening to others' such as clients' similar stories. KB-DSS based on narrative-reasoning can equip decision makers to better adapt to existing experience and discover more innovative ideas to solve new decision problems [13].

Most exiting work on KB-DSS has focused on the technology perspective such as IT technology, in particular the AI and computer linguistics, to support knowledge representation, reasoning and process to develop knowledge management systems. Less effort has been committed to understand the knowledge management requirements from business decision makers, for example, business knowledge acquisition and structuring, knowledge re-use, knowledge mobilization, critical knowledge, knowledge chain management, and knowledge lifecycle from organizational, social and cultural perspectives. Current KB-DSS has been criticized for a number of limitations, for example, the static nature of the knowledge base; difficulty of re-using knowledge; knowledge is understood from single point of view, such as many scholars simply referred knowledge as "know-how"; users lose confidence in knowledge because of errors, obsolescent and unnecessary knowledge that make the knowledge base crowded, messy and fat; lack of knowledge refinement resulting in unsure about knowledge performance; human-computer interface not intelligent enough to allow 
two-way conversations between decision makers and KB-DSS. Such gaps in the literature have motivated a number of projects undertaken by the authors in recent years with a focus on KB-DSS in contemporary business management decision context, trying to overcome the limitations. The next section discusses the contribution to new knowledge from these research projects.

\section{KEY PERSPECTIVES OF KB-DSS FROM RECENT RESEARCH PROJECTS}

This section presents the results of six research projects recently undertaken by the authors to address the knowledge support issue for DSS from different perspectives. The projects made significant contribution to new knowledge in KB-DSS, in particular in terms of knowledge acquisition and structuring, knowledge reuse, knowledge mobilization, critical knowledge identification, knowledge chain management, and knowledge integration.

\section{A. Project 1: Knowledge Levels and Structuring}

How to better capture domain expert's knowledge and structure it in the knowledge base has been a long time ongoing research topic. It seems to have two crucial questions. One is how many levels should knowledge be defined. The other question is how many knowledge components or segments should be retained in a knowledge base. Structuring knowledge base in how many segments or components often depends on the nature of the domain knowledge. These two questions have been investigated through a project which was focused on developing a KB-DSS for lean production management. In order to consider both knowledge level and knowledge component dimensions, the project created a knowledge acquisition and structuring model which defines four levels of knowledge and seven knowledge components [14]. A diagrammatic illustration of the knowledge matrix is shown in Fig. 2. Literature has widely discussed the "know-what" (i.e. declarative knowledge) and "know-how" (i.e. procedural knowledge) levels of knowledge in various knowledge management scenarios. The contribution of the project is not only to have investigated the "know-what" and "know-how" in lean production management decision making, but also implemented two new levels of knowledge , i.e. "know-why" and "know-with" in the KB-DSS. One advantage of implementing "know-why" in the KB-DSS is that the systems can provide principles underlying "know-how" and "know-what" for decision justification. The "know-with" specifies the interrelationships among knowledge components for integrated decision support [14].

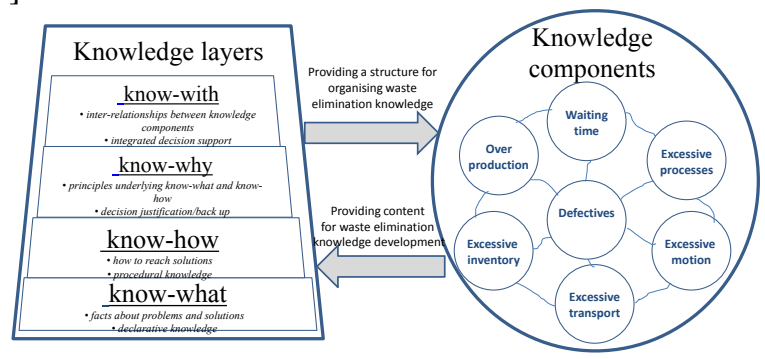

Fig. 2. Four levels of knowledge [14].

\section{B. Project 2: Knowledge Reuse}

Knowledge re-use in of course one of the main reasons why KBS are developed in the first place. In terms of decision support for business management, decision makers can always make use of the existing knowledge in the knowledge base to get better understanding of the market, customers, resources, processes, and quantity control. If the decision makers are novices in the business domain, through re-using external knowledge of their peers, it is more likely for them to have the chance to make the right business decisions about products and services to create, supply chain resources to recruit, marketing channels to explore, and investment choices to gain maximum profit for the companies. For expert decision makers, through knowledge re-use, they can reapply proven solutions, learn from use and failures, avoid pitfalls and increase the chances to make the right decisions over time. There has been wide interest in knowledge re-use research. One of the widely cited piece of work published by Markus defined four types of users who can benefit from knowledge-reuse, including shared work producers (they create the knowledge themselves and later reuse the knowledge), shared work practitioners (they do not create knowledge but reuse knowledge created by their co-workers), knowledge miners, and expertise seeking novices [15]. A project undertaken by the authors developed a structural knowledge re-use model which contains four more key components in addition to Markus's re-user types and specifies the relationships between all five key components [3]. The four extra components are Knowledge Types (such as best practice, lessons learnt, rational knowledge, procedural knowledge), Knowledge Sources (e.g. repositories, systems, individual records), Knowledge Environment (including model environment, system environment, document environment, and knowledge base environment), and Knowledge Integration Approaches (i.e. network-based, traceability-based, and ontology-based). The UML representation of the structural knowledge re-use model is shown in Fig. 3.

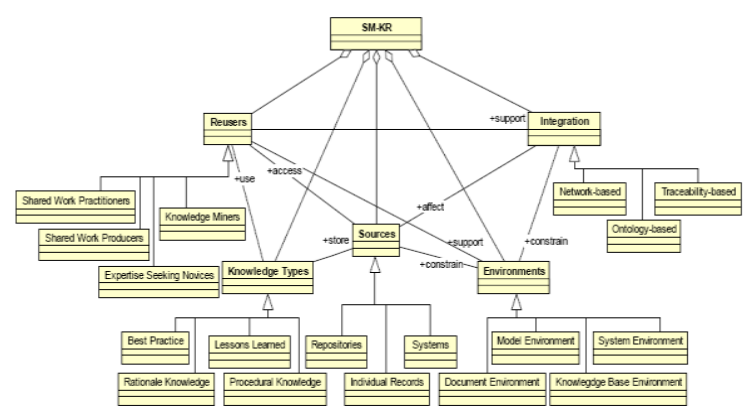

Fig. 3. Knowledge re-use model in UML representation.

\section{Project 3: Knowledge Mobilization}

Investigating knowledge mobilization for decision support is a necessity when organizations are going through changes or when knowledge needs to be shared, transferred, translated (because of culture difference) or disseminated in communities or across supply chain partners. There are many factors that could affect knowledge mobilization, including organizational culture, organizational strategy, organizational capacity and knowledge infrastructure. In 
order to enable knowledge mobilization, it is important to establish knowledge networks. A project currently being undertaken by the authors has created a knowledge mobilization model to support decision making in IT project change management [16]. The knowledge mobilization model defines connections between four types of knowledge networks, including the knowledge networks of interaction, knowledge networks of interpretation and translation, knowledge networks of influence, and the institutional knowledge networks (i.e. the knowledge base). The knowledge mobilization model is centred around the classic SECI (socialization, externalization, combination and internalization) model proposed by Nonaka \& Takeuchi [17]. By doing so, there is a potential that the right knowledge can be transferred in the right format to the right people to support them make the right decisions. Fig. 4 illustrates the knowledge mobilization model.

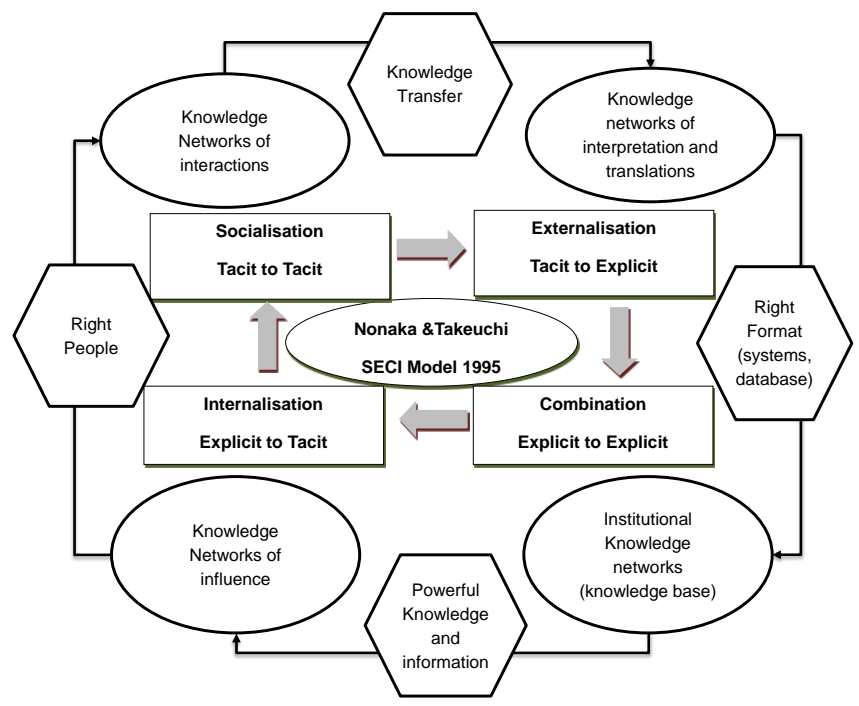

Fig. 4. A knowledge mobilization model.

\section{Project 4: Critical Knowledge}

How much knowledge should be stored in the knowledge base of a KB-DSS has remained as a key question under investigation. It is easy to understand that keeping unnecessary knowledge in the knowledge base will not only cause waste of resource to hold and update the knowledge base, but also cause distraction and confusion when users try to query and retrieve the required knowledge from the knowledge base. Identifying critical knowledge is a relatively new topic and there has not been a concerted definition of what critical knowledge is. Many scholars have defined critical knowledge in various ways, for example, as "the necessary knowledge to solve problems dealing with a given objective and that should be capitalized" [18], "essential that contributes to added value and business performance" [19], "vital expertise, ideas and insights" [20], and "with regard to its scarcity, cost and delay of acquisition" [21]. The authors" current project develops a lean knowledge model that only contains critical knowledge to optimise the knowledge inventory for supply chain management decisions. The main ideas of the lean knowledge model are shown in Fig. 5. It is proposed that critical knowledge can be identified through systematic processes such as using GAMETH method [22].
The identified critical knowledge can however still flow in and out of the knowledge base all the time. Two strategies can be used to optimize the knowledge inventory level, i.e. just in time (JIT) and just in case (JIC) strategies, in order to sustain a lean knowledge base [23].

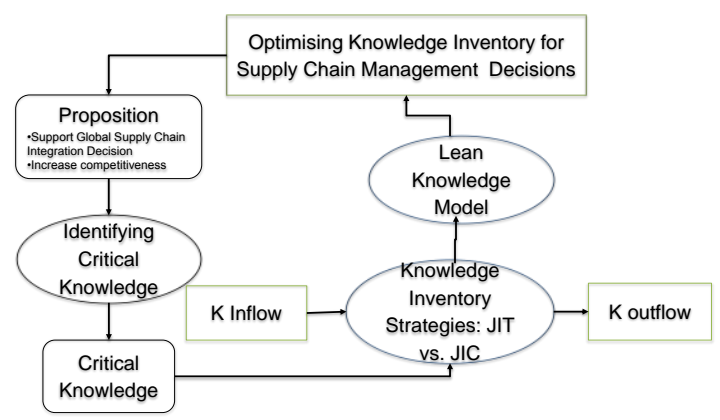

Fig. 5. Critical knowledge in lean knowledge model.

\section{E. Project 5: Knowledge Chain Management}

When developing KB-DSS for supply chain management, knowledge should not be seen as a static entity in the knowledge repository, but as a dynamic process because knowledge can change and evolve when it flows along supply chains. Just as products and materials flow along supply chains, knowledge chain has to be created and maintained so that knowledge flow through supply chain partners can be as smooth as possible. The earliest knowledge chain model was only proposed at the beginning of 21 century which includes five primary knowledge activities and four secondary knowledge activities, as shown in Fig. 6 [24]. Authors' research extended Holsapple and Singh's knowledge chain model to enable decision support in global supply chain management context by adding three new knowledge chains to include global customer knowledge, global capacity knowledge, and global supply network configuration knowledge [25].

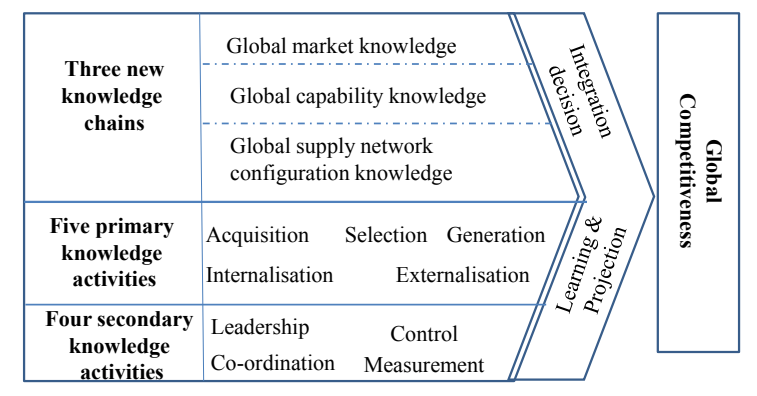

Fig. 6. Knowledge chain model to support decision making in global supply chain management.

\section{F. Project 6: Knowledge Integration}

Knowledge integration has been recognized as a key issue in all knowledge based systems, and KB-DSS is no exception. A lot of scholars have put efforts in knowledge integration, but so far progress still remains in conceptual models and lacks mature software tools that can be readily integrated in KB-DSS. The authors have explored an integrative framework for knowledge management in support of ERP (Enterprise Resource Planning) systems implementation 
decisions. The framework recommends that knowledge management should look at the whole knowledge life cycle together with the types of knowledge and multiple levels of knowledge, as illustrated in Fig. 7 [26]. The knowledge lifecycle includes four stages: knowledge creation, knowledge retention, knowledge transfer and knowledge application. Specific knowledge types investigated in the project are ERP package knowledge, organizational culture knowledge, business process knowledge and project management knowledge. The four levels of knowledge specified in project 1 are explored here in a different decision context, i.e. ERP implementation, they are know-what, know-how, know-why and know-with. More importantly, this project focused on the exploration of the links across the knowledge types, knowledge layers and knowledge lifecycle.

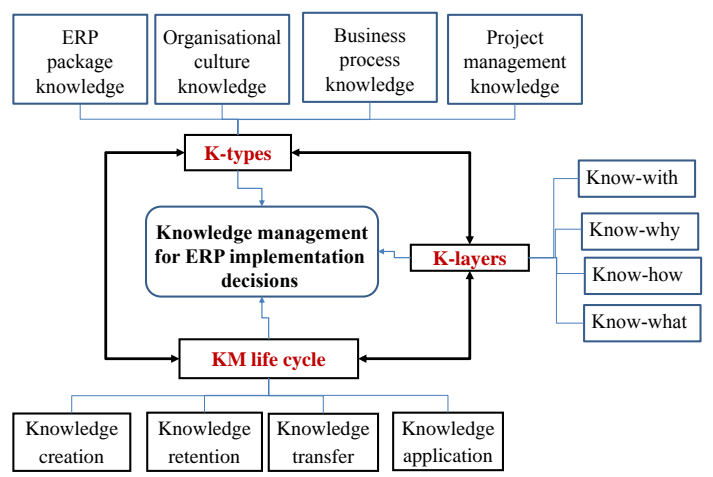

Fig. 7. An integrative knowledge framework for ERP implementation decisions.

\section{IMPLICATIONS AND PROPOSED NEW ARCHITECTURE FOR FUTURE WORK}

Based on the outputs from the six projects undertaken by the authors, we have learnt that knowledge management in KB-DSS is multi-faceted and requires researchers' greater endeavor to gain insightful understanding of how KB-DSS can be best used to improve decision maker's decision performance in real business world. However, some implications can be highlighted for the development of more intelligent KB-DSS:

1) Knowledge should not be seen as a static entity in the knowledge base, but process-oriented as knowledge evolves with the decision environment in organization, society and culture.

2) Knowledge needs to be shared and re-used to accommodate users' different roles in different decision situations.

3) Decision support needs multi-level of knowledge, i.e. besides know-what and know-how, KB-DSS should provide know-why for decision justification and know-with for integrated decision support.

4) KB-DSS needs to consider knowledge validation and evaluation to check the knowledge credibility and criticality, to make sure the knowledge held in the knowledge base is correct, current and that the knowledge base is lean.

5) Knowledge held in KB-DSS needs to be refined according to knowledge relationships, knowledge performance and evolving business performance objectives.

6) KB-DSS needs intelligent HCI so that decision makers can use the systems to take better control of the decision situations, define and specify the decision problems, direct the decision processes, inquire and question the systems more easily, and make improved judgment, while the KB-DSS can better adapt to changing decision situations, evolve with decision environment, explain better about the rationale behind recommendations, guide the decision makers to the right choices, learn from the decision makers, suggest solutions and answer decision maker's queries. That means that KB-DSS can act more than just an assistant but also a mentor and an advisor which can stimulate decision maker's innovative ideas and critical thinking.

To knit the ideas together for the development of a potentially improved KB-DSS, a new architecture is proposed for future work, as illustrated in Fig. 8. It can be seen from the Fig. 8, the knowledge management sub-system in KB-DSS will have five new components in addition to the existing knowledge base and inference engine (with reasoning mechanisms). A meta-knowledge component will allow the KB-DSS to implement multi-levels of knowledge to include know-why and know-with on top of know-what and know-how. A knowledge validation/ evaluation component will allow the check for knowledge correctness, precision, credibility and criticality. The knowledge refinement component can imitate human experts so as to analyze their knowledge and its effectiveness, learn from it, and improve on it for future decision consultations. The KB-DSS will be able to create user profiles based on their knowledge to capture the user's role in decision making processes, their expertise level and their behavior in using the systems over time. The knowledge traceability component will facilitate knowledge mobilization and knowledge integration when dealing with decision situations across organizations and supply chains. In order to evaluate this new architecture, future work would be implementing the system and applying the system to various business decision cases.

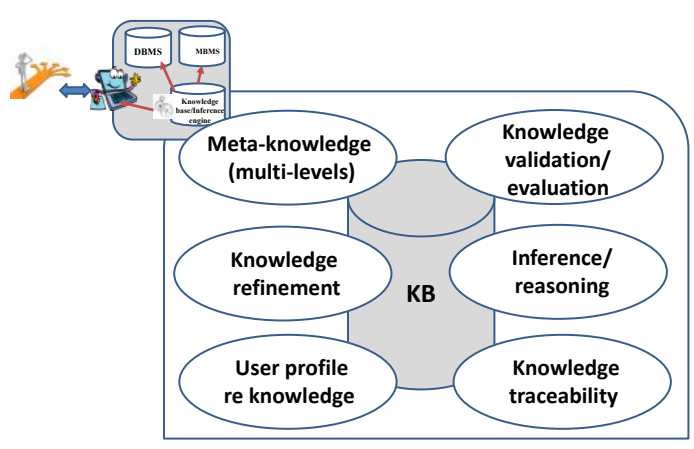

Fig. 8. A new architecture for future KB-DSS

\section{CONCLUSIONS}

Knowledge-based decision support systems (KB-DSS) have been investigated for nearly three decades and have supported real business management decisions in many industries. It has been recognized that KB-DSS have improved decision maker's performance, especially in terms of speed and consistency. This paper presents new 
contributions from six research projects undertaken by the authors to further improve the knowledge management function in KB-DSS with respect to knowledge levels and structuring, knowledge re-use, knowledge mobilization, critical knowledge, knowledge chain management and knowledge integration. A new architecture is proposed for future work in developing more intelligent KB-DSS.

\section{ACKNOWLEDGMENT}

The authors would like to thank a number of parties which have funded the work presented in this paper, including UK Engineering and Physical Science Research Council, University of Plymouth and Saudi Arabia government.

\section{REFERENCES}

[1] P. Zarate, Tools for Collaborative Decision Making, Wiley, ISBN: 978-1-84821-516-0, 2013.

[2] C. Carlsson and E. Turban, "DSS: directions for the next decade," Decision Support Systems, vol. 33, pp. 105-110, Jun 2002.

[3] S. Liu, A. H. B. Duffy, I. M. Boyle, and R. I. Whitfield, "Knowledge re-use for decision support," in Proc. Realizing Network Enabled Capability, Leeds, UK, 2008, pp. 18-26.

[4] S. Liu, A. H. B. Duffy, R. I. Whitfield, and I. M. Boyle, "Integration of decision support systems to improve decision support performance," Knowledge and Information Systems, vol. 22, issue. 3, pp. 261-286, Jan 2010.

[5] R. H. Sprague and E. D. Carlson, Building Effective Decision Support Systems, Englewood Cliffs, N. J., Prentice-Hall, 1982.

[6] C. W. Holsapple and K. D. Joshi, "Organizational knowledge resources," Decision Support Systems, vol. 31, no. 4, pp. 39-54, May 2001.

[7] G. M. Marakas, Decision Support Systems in the $21^{\text {st }}$ Century, $2^{\text {nd }}$ edition. Pearson Education, 2003.

[8] M. Alvarado, M. A. R. Toral, A. Rosas, and S. Ayala, "Decision making on pipe stress analysis enabled by knowledge-based systems," Knowledge and Information Systems - an International Journal, vol. 12, no. 2, pp. 255-278, Jun 2007.

[9] R. A. Akerkar and P. S. Sajja, Knowledge-Based Systems, Jones and Bartlett Publishers, Boston, USA. 2010.

[10] G. Kong, D. L. Xu, R. Body, J. B. Yang, K. M. Jones, and S. Carley, “A belief rule-based decision support system for clinical risk management of cardiac chest pain," European Journal of Operational Research, vol 219 , pp. 564-573, 2012.

[11] I. Bichindaritz and S. Montani, "Advances in case-based reasoning in the health sciences," Artificial Intelligence in Medicine, vol. 51, pp. 75-79, 2011.

[12] E. J. M. Lauria and P. J. Duchessi, "A Bayesian belief network for IT implementation decision support," Decision Support Systems, vol. 42 , pp. 1573-1588, Dec 2006.

[13] W. M. Wang and C. F. Chueng, "A narrative-based reasoning with applications in decision support for social service organisations," Expert Systems with Application, vol. 38, pp. 3336-3345, Apr. 2011.

[14] S. Liu, F. Annansingh, J. Moizer, L. Liu, and W. Sun, "A knowledge system for integrated production Waste Elimination in support of organisational decision making," in Lecture Notes in Business Information Processing, J. E. Hernandez, P. Zarate, F. Dargam, B. Delibasic, S. Liu, and R. Ribeiro, Ed. Springer, 2012, vol. 121, pp. 134-150.

[15] M. L. Markus, "Toward a theory of knowledge re-use: types of knowledge re-use situations and factors in re-use success," Journal of Management Information Systems, vol. 18, pp. 57-91, 2011.

[16] A. Alkuraiji, S. Liu, F. Annansingh, and J. Pang, "Knowledge network modelling to support decision making for strategic intervention in IT Project-oriented change management," presented at the $26^{\text {th }}$ Joint International Conference between EURO-INFORMS, Rome, Italy, 1-4 July 2013.

[17] I. Nonaka and H. Takeuchi, "The knowledge creating company: How Japanese companies create the dynamics of innovation," New York: Oxford University Press, 1995.

[18] T. Tseng and C. Huang, "Capitalizing on knowledge: a novel approach to crucial knowledge determination," IEEE Transactions on Systems, Man, and Cybernetics-Part A: Systems and Humans, vol. 25, no. 6 , 2005.
[19] M. Grundstein, C. R. Sabroux, and A. Pachulski, "Reinforcing decision aid by capitalizing on company's knowledge," European Journal of Operational Research, vol. 145, pp. 256-272, 2003.

[20] S. Y. Huang and J. N. Cummings, "When critical knowledge is most critical centralization in knowledge-intensive team," Small Group Research, vol. 42, pp. 669-699.

[21] J. Ermine, I. Boughzaka, and T. Tounkara, "Critical knowledge map as a decision tool for knowledge transfer actions," The Electronic Journal of Knowledge Management, vol. 4, iss. 2, pp. 129-140, Apr 2006.

[22] M. Grundstein and C. Rosenthal-Sabroux. (2005). A process modelling approach to identify and locate potential crucial knowledge: the CAMETH framework. [Online]. Accessed http://basepub.dauphine.fr/xmlui/bitstream/handle/123456789/4135/I RMA05(GAMETH)text-1.pdf? sequence=2

[23] J. Pang, S. Liu, S. Tuck, and A. Alkuraiji, "Optimising inventory level of global critical knowledge for integrated decision support," presented at the $26^{\text {th }}$ Joint International Conference between EURO-INFORMS, Rome, Italy, 1-4 July 2013.

[24] C. W. Holsapple and M. Singh, "The knowledge chain model: activities for competitiveness," Expert Systems with Applications, vol. 20, pp. 77-98, Jan 2001

[25] S. Liu, J. Moizer, P. Megicks, D. Kasturiratne, and U. Jayawickrama, "A knowledge chain management framework for global supply chain integration decisions" Production Planning and Contro, 2013.

[26] U. Jayawickrama, S. Liu, and M. H. Smith, "An integrative knowledge management framework to support ERP implementation for improved management decision making in industry," in Lecture Notes in Business Information Processing, J. E. Hernandez, S. Liu, B. Delibasic, P. Zarate, F. Dargam, and R. Ribeiro, Ed. Springer, 2013, vol. 164, pp. 86-101.

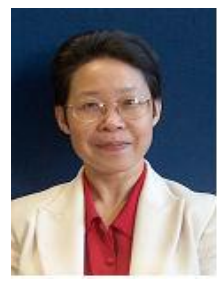

Shaofeng Liu is a professor of Operations Management and Decision Making at the University of Plymouth, UK. She obtained her Ph.D. degree from Loughborough University, UK, specialising in Knowledge and Information Management for Global Manufacturing Co-ordination Decisions. Her main research interests and expertise are in knowledge-based techniques to support business decision making, particularly in the areas of knowledge management, integrated decision support, ERP systems and quantitative decision methods for lean operations, process improvement, resource management, quality management, and supply chain management. She is currently supervising 7 $\mathrm{PhD}$ students in above research areas. She has undertaken a number of influential research projects funded by UK research councils and the European Commission. She has published over 90 peer-reviewed research papers including 50 journal articles, 5 book chapters, 23 conference papers, and editorial for 6 journal Special Issues and 5 conference/workshop proceedings. She is currently an associate editor for the Journal of Decision Systems and on the Editorial Board for the International Journal of Decision Support Systems Technology. She conducts regular review for 3 research councils and 10 international journals.

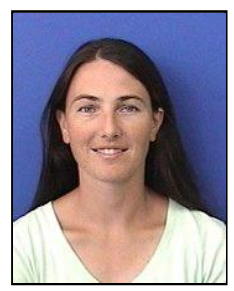

Melanie Hudson Smith is a lecturer in Operations Management at the University of Plymouth. Her primary research interests are in operations improvement, sustainable supply chains and service quality, with recent publications in these areas. She teaches Operations at both undergraduate and postgraduate levels and supervises a number of Ph.D. students.

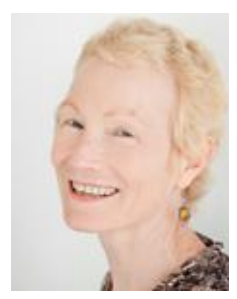

Sarah Jane Tuck spent 12 years at sea as a deck officer where she qualified as a master mariner. She also served for a time in the royal naval reserve. She undertook her Ph.D. at Plymouth University, examining the socio-economics of small ports and wharves in the southwest of England. She is presently a lecturer in maritime business within the International Shipping, Logistics \& Operations group at Plymouth University. Her research interests include sustainable ports and shipping, qualitative research methods and short sea shipping. Her research grants include a successful Knowledge Transfer Partnerships (KTP) project with Falmouth Harbour Commissioners, and the Low Carbon Shipping Consortium, both funded by the UK government. 


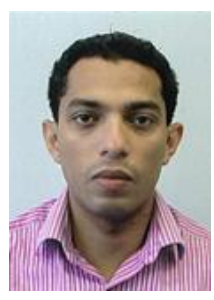

Ali Alkhuraiji is a researcher at Graduate School of Management, University of Plymouth, UK. He was awarded an MSc degree by Loughborough

University, UK in 2011, specialising in Information

Science, in particular in information and knowledge management. His area of research is on knowledge management, IT projects, decision making and knowledge mobilization. He has presented some of his research at International conference held in Rome, Italy at the 2013 Euro Conference

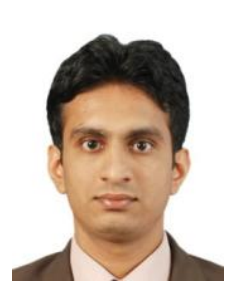

Uchitha Jayawickrama is currently a doctoral researcher at Plymouth Business School, University of Plymouth, United Kingdom. He worked as an Oracle Applications Consultant for Oracle E-Business Suite (ERP) systems implementation in various industries $\mathrm{He}$ is an MBA holder from University of Sri Jayewardenepura, Sri Lanka. He obtained his BSc. honours degree in Information Systems from Sri Lanka Institute of Information Technology (SLIIT), Sri Lanka. His main research and teaching interests are in ERP systems, knowledge management, decision making and information systems. He has published research papers in journals and presented his research studies at different international conferences. 